\title{
Stability Analysis for a Class of Discrete-Time Nonhomogeneous Markov Jump Systems with Multiplicative Noises
}

\author{
Shaowei Zhou $\left(\mathbb{D},{ }^{1,2}\right.$ Xiaoping Liu, ${ }^{3,4}$ Bing Chen, ${ }^{2}$ and Hongxia Liu ${ }^{1}$ \\ ${ }^{1}$ College of Mathematics and Systems Science, Shandong University of Science and Technology, Qingdao 266590, China \\ ${ }^{2}$ Institute of Complexity Science, Qingdao University, Qingdao 266071, China \\ ${ }^{3}$ Department of Electrical Engineering, Lakehead University, Thunder Bay, ON, Canada P7B 5E1 \\ ${ }^{4}$ College of Information and Electrical Engineering, Shandong Jianzhu University, Jinan 250101, China
}

Correspondence should be addressed to Shaowei Zhou; zsw9675@163.com

Received 12 July 2017; Revised 17 December 2017; Accepted 9 January 2018; Published 25 February 2018

Academic Editor: Michele Scarpiniti

Copyright (c) 2018 Shaowei Zhou et al. This is an open access article distributed under the Creative Commons Attribution License, which permits unrestricted use, distribution, and reproduction in any medium, provided the original work is properly cited.

\begin{abstract}
This paper is concerned with a class of discrete-time nonhomogeneous Markov jump systems with multiplicative noises and timevarying transition probability matrices which are valued on a convex polytope. The stochastic stability and finite-time stability are considered. Some stability criteria including infinite matrix inequalities are obtained by parameter-dependent Lyapunov function. Furthermore, infinite matrix inequalities are converted into finite linear matrix inequalities (LMIs) via a set of slack matrices. Finally, two numerical examples are given to demonstrate the validity of the proposed theoretical methods.
\end{abstract}

\section{Introduction}

There are a lot of dynamic systems which are subjected to abrupt variations in parameters caused by external surroundings or internal structure in practical engineering field. Markov jump system models have attracted more and more attention because they effectively described this kind of systems and have become a hot topic in control theory. Many mature and systematic results have been obtained [1-5].

It should be pointed out that most of the current research is done in the framework of homogeneous Markov process (or Markov chain), which is under the assumption that the transition rate (or transition probability) matrix of the system at any time is the same. In fact, due to the impact of the objective environment, this assumption is difficult to satisfy. For example, in a Markov jump networked control system, the transition probability is time-variant because the packet dropouts and network delays are different at different periods. Another typical example can be found in the faultprone systems, where Markov process is used to describe the failure rate that is influenced by the factors of the age and the usage rate. Obviously, it is not a homogeneous process. Driven by these practical problems, people turn to the nonhomogeneous Markov jump systems [6-21].

In order to describe the nonhomogeneous property, several assumptions are put forward. In [6], the time-varying transition probabilities of discrete-time Markov jump systems are considered to be finite piecewise homogeneous with two types of variations in the finite set: arbitrary variation and stochastic variation, which implies that the transition probabilities are varying but invariant within an interval. The $H_{\infty}$ estimation problem is investigated. This assumption is generalized to the continuous-time Markov jump system [7], Markov jump neural networks [8,9], complex networks [10], and singular Markov jump systems [11], and the robust stability, stochastic stability, passivity analysis, and synchronization are studied, respectively. Another way of describing timevarying characteristics is in a polytopic sense. It is proposed for the first time in [12] and a sufficient condition for stochastic stability is derived by using a parameter-dependent stochastic Lyapunov function. The main idea is that the transition probability matrix is assumed to be valued in a polytope convex set with some given vertices when the exact transition probability is not known. The polytopic model 
is more general and includes the piecewise homogeneous Markov jump system model with arbitrary switch as a special case. Subsequently, many new results are obtained on this model. In [13-15], mode-dependent, variation-dependent, and observer-based $H_{\infty}$ controllers are designed to satisfy the stochastic stability and prescribed $H_{\infty}$ performance index. The other control problems, such as $l_{2}-l_{\infty}$ control [16] and fault detection [17], are also considered. An application to DC motors can be found in [18]. The model is used not only in discrete-time systems but also in continuous-time systems; see [19]. However, up to now, the model has not been applied to Markov jump systems with multiplicative noises by the authors' knowledge. This kind of systems with multiplicative noises is often used in engineering practice. For example, a practical model with the control input dependent noise in CDMA systems can be found in [22]. This note aims to make an attempt to investigate the stability of such systems.

Our purpose is to address the stability analysis and stabilization controller design for discrete-time nonhomogeneous Markov jump systems with multiplicative noises and with polytopic transition probability matrices. It is well known that stability analysis is the basis for the design and synthesis in all control systems and fruitful results have been obtained; see [23-30] and the references therein. In this article, two types of stability, stochastic stability (SS) in mean square sense and finite-time stability (FTS), will be considered.

The organization of this paper is as follows. In Section 2, we provide model formulation and give some definitions. Section 3 is devoted to stochastic stability and stabilization. In Section 4, the finite-time stability and stabilization will be discussed. Some numerical examples are presented in Section 5. Finally, a brief concluding remark is given in Section 6

Notations 1. $\mathbf{R}^{n}$ is $n$-dimensional Euclidean space; $A^{\mathrm{T}}$ is the transpose of matrix $A ;|A|$ is the determinant of matrix $A$; $A>0: A$ is a positive definite matrix; $\mathbf{I}$ is the identity matrix; $\|x\|$ is the Euclidean norm of vector $x ; E(\cdot)$ is the expectation operator; $\delta_{(\cdot)}$ is Kronecker function; $\operatorname{diag}\left(A_{1}, \ldots, A_{n}\right)$ is a block diagonal matrix with the block diagonal elements $A_{1}, \ldots, A_{n} ; \lambda_{\min }(A)\left(\lambda_{\max }(A)\right)$ is the minimum (maximum) eigenvalue of matrix $A$; $\operatorname{Cond}(A)$ is the condition number of matrix $A$; * is the symmetric part of a symmetric matrix; $\mathbf{N}_{0}=\{0,1,2, \ldots\}, \mathbf{N}=\{1,2, \ldots\}$, and $\mathbf{N}_{T}=\{1,2, \ldots, T\}$.

\section{Problem Formulation}

Consider the following discrete-time stochastic Markov jump system with multiplicative noises:

$$
\begin{aligned}
& x_{k+1}=A\left(r_{k}\right) x_{k}+C\left(r_{k}\right) x_{k} w_{k}, \\
& x(0)=x_{0} .
\end{aligned}
$$

And the corresponding controlled system is as follows:

$$
\begin{aligned}
x_{k+1}= & A\left(r_{k}\right) x_{k}+B\left(r_{k}\right) u_{k} \\
& +\left[C\left(r_{k}\right) x_{k}+D\left(r_{k}\right) u_{k}\right] w_{k}, \\
x(0)= & x_{0}
\end{aligned}
$$

where $x_{k} \in \mathbf{R}^{n}$ and $u_{k} \in \mathbf{R}^{m}$ are the system state and control input, respectively. $x_{0} \in \mathbf{R}^{n}$ is the initial state and $A(\cdot)$, $B(\cdot), C(\cdot)$, and $D(\cdot)$ are real coefficient matrices with the appropriate dimension. $\left\{w_{k}, k \in \mathbf{N}_{0}\right\}$ is a sequence of real random variables defined on a complete probability space $(\Omega, \mathscr{F}, P)$ with $E\left(w_{k}\right)=0$ and $E\left(w_{s} w_{t}\right)=\delta_{(s-t)}$. The jump process $\left\{r_{k}, k \in \mathbf{N}_{0}\right\}$ is described by a discrete-time nonhomogeneous Markov chain which takes values in a finite set $S=\{1,2, \ldots, s\}$. The transition probability is $\pi_{i j}(k)=$ $P\left(r_{k+1}=j \mid r_{k}=i\right)$ which means the probability from mode $i$ at time $k$ to mode $j$ at time $k+1$ and satisfies $\pi_{i j}(k) \geq 0$, $\sum_{j=1}^{s} \pi_{i j}(k)=1$. The transition probability matrix is $\Gamma(k)=$ $\left[\pi_{i j}(k)\right]$. It is assumed that $\Gamma(k)$ is time-varying and takes values in a polytope with its vertices $\Gamma^{l}, l=1,2, \ldots, m$; that is,

$$
\begin{aligned}
\Gamma(k) & =\sum_{l=1}^{m} \alpha_{l}(k) \Gamma^{l}, \quad \alpha_{l}(k) \geq 0, \\
\sum_{l=1}^{m} \alpha_{l}(k) & =1
\end{aligned}
$$

where $\Gamma^{l}(l=1,2, \ldots, m)$ are given matrices and the entries of $\Gamma^{l}$ are written by $\pi_{i j}^{l} .\left\{w_{k}, k \in \mathbf{N}_{0}\right\}$ is independent of $\left\{r_{k}, k \in\right.$ $\mathbf{N}_{0}$.

To be convenient, we denote the coefficient matrices associated with $r_{k}=i$ as $M_{i}=M\left(r_{k}\right)$.

In this paper, we mainly formulate some conditions of SS and FTS. They are two different and independent stability concepts: SS describes the asymptotic behavior of the systems in infinite time domain, while FTS reflects transient performance of the systems in finite-time interval. Now, let us recall the definitions.

Definition 2 (see [1]). System (1) is called stochastically stable if for any initial state $x_{0}$ and initial mode $r_{0}$,

$$
\sum_{k=0}^{\infty} E\left(\left\|x_{k}\right\|^{2}\right)<\infty .
$$

System (2) is called stochastically stabilizable if there exist a sequence of feedback controls $u_{k}=K\left(r_{k}\right) x_{k}$ such that for any initial state $x_{0}$ and initial mode $r_{0}$, the closed-loop system

$$
\begin{aligned}
x_{k+1}= & {\left[A\left(r_{k}\right)+B\left(r_{k}\right) K\left(r_{k}\right)\right] x_{k} } \\
& +\left[C\left(r_{k}\right)+D\left(r_{k}\right) K\left(r_{k}\right)\right] x_{k} w_{k}, \\
x(0)= & x_{0}
\end{aligned}
$$

is stochastically stable.

Definition 3 (see [23]). System (1) is called finite-time stable with respect to $\left(c_{1}, c_{2}, R, T\right)$, if

$$
E\left(x_{0}^{\mathrm{T}} R x_{0}\right) \leq c_{1} \Longrightarrow E\left(x_{k}^{\mathrm{T}} R x_{k}\right) \leq c_{2}, \quad \forall k \in N_{T},
$$

where $R>0$ is a given matrix, $c_{2}>c_{1}>0$, and $T \in \mathbf{N}$ are the given real numbers. System (2) is said to be finitetime stabilizable if there exist a sequence of feedback controls $u_{k}=K\left(r_{k}\right) x_{k}$ such that the closed-loop system (5) is finitetime stable. 


\section{Stochastic Stability and Stabilization}

In this section, some sufficient conditions will be provided for the stochastic stability and stabilization of systems (1) and (2).

Theorem 4. If there exist a set of matrices $P_{i}^{l}>0, \forall i \in$ $S, l=1,2, \ldots, m$, such that for every $\alpha_{l}(k)$ and $\beta_{n}(k), l, n=$ $1,2, \ldots, m$,

$$
\begin{aligned}
& A_{i}^{\mathrm{T}}\left(\sum_{j=1}^{s} \sum_{l=1}^{m} \sum_{n=1}^{m} \alpha_{l}(k) \beta_{n}(k) \pi_{i j}^{l} P_{j}^{n}\right) A_{i} \\
& \quad+C_{i}^{\mathrm{T}}\left(\sum_{j=1}^{s} \sum_{l=1}^{m} \sum_{n=1}^{m} \alpha_{l}(k) \beta_{n}(k) \pi_{i j}^{l} P_{j}^{n}\right) C_{i} \\
& \quad-\sum_{l=1}^{m} \alpha_{l}(k) P_{i}^{l}<0,
\end{aligned}
$$

where $0 \leq \alpha_{l}(k), \beta_{n}(k) \leq 1, \sum_{l=1}^{m} \alpha_{l}(k)=1$, and $\sum_{n=1}^{m} \beta_{n}(k)=$ 1 , then system (1) is stochastically stable.

Proof. Let $V\left(x_{k}, r_{k}=i\right)=x_{k}^{\mathrm{T}}\left(\sum_{l=1}^{m} \alpha_{l}(k) P_{i}^{l}\right) x_{k}$ and $\mathscr{F}_{k}$ be the $\sigma$-algebra generated by $\left\{\left(r_{t}, x_{t}\right), t=0,1, \ldots, k\right\}$. Then,

$$
\begin{aligned}
\triangle & V\left(x_{k}, r_{k}=i\right)=E\left[V\left(x_{k+1}, r_{k+1}=j\right)\right]-V\left(x_{k}, r_{k}=i\right) \\
& =E\left[E\left(x_{k+1}^{\mathrm{T}} \sum_{l=1}^{m} \alpha_{l}(k+1) P_{j}^{l} x_{k+1} \mid \mathscr{F}_{k}\right)\right] \\
& =E\left[x_{k+1}^{\mathrm{T}}\left(\sum_{j=1}^{s}\left(\sum_{l=1}^{m} \alpha_{l}(k+1) P_{j}^{l} P\left(r_{k+1}=j \mid r_{k}=i\right)\right)\right)\right. \\
\cdot & \left.x_{k+1}\right]-x_{k}^{\mathrm{T}}\left(\sum_{l=1}^{m} \alpha_{l}(k) P_{i}^{l}\right) x_{k} \\
= & {\left[x_{k+1}^{\mathrm{T}}\left(\sum_{j=1}^{s}\left(\pi_{i j}(k) \sum_{l=1}^{m} \alpha_{l}(k+1) P_{j}^{l}\right)\right) x_{k+1}\right] } \\
& -x_{k}^{\mathrm{T}}\left(\sum_{l=1}^{m} \alpha_{l}(k) P_{i}^{l}\right) x_{k}=E\left[x _ { k + 1 } ^ { \mathrm { T } } \left(\sum_{j=1}^{s}\left(\sum_{l=1}^{m} \alpha_{l}(k) \pi_{i j}^{l}\right)\right.\right. \\
& =x_{k}^{\mathrm{T}}\left(\sum_{l=1}^{m} \alpha_{l}^{\mathrm{T}}(k) P_{i}^{l}\right) x_{k} \\
& \left.\left.\cdot\left(\sum_{n=1}^{m} \beta_{n}(k) P_{j}^{l}\right)\right) x_{k+1}\right]-x_{k}^{\mathrm{T}}\left(\sum_{l=1}^{m} \alpha_{l}(k) P_{i}^{l} \sum_{l=1}^{m} \sum_{n=1}^{m} \alpha_{l}(k) \beta_{n}(k) \pi_{i j}^{l} P_{j}^{l}\right) A_{i} \\
& {\left[x_{k+1}^{\mathrm{T}}\left(\sum_{j=1}^{s} \sum_{l=1}^{m} \sum_{n=1}^{m} \alpha_{l}(k) \beta_{n}(k) \pi_{i j}^{l} P_{j}^{l}\right) x_{k+1}\right] }
\end{aligned}
$$

$$
\begin{aligned}
& \left.+C_{i}^{\mathrm{T}}\left(\sum_{j=1}^{s} \sum_{l=1}^{m} \sum_{n=1}^{m} \alpha_{l}(k) \beta_{n}(k) \pi_{i j}^{l} P_{j}^{l}\right) C_{i}-\sum_{l=1}^{m} \alpha_{l}(k) P_{i}^{l}\right] \\
& \cdot x_{k} \triangleq x_{k}^{\mathrm{T}} \Psi_{i}(k) x_{k} .
\end{aligned}
$$

So we have

$$
\begin{aligned}
\triangle V\left(x_{k}, r_{k}=i\right) & \leq-\min _{i \in S}\left\{\min _{k \in \mathbf{N}_{0}}\left\{\lambda_{\min }\left(-\Psi_{i}(k)\right)\right\}\right\} x_{k}^{\mathrm{T}} x_{k} \\
& \triangleq-\rho x_{k}^{\mathrm{T}} x_{k}
\end{aligned}
$$

obviously, $\rho>0$; thus

$$
\begin{aligned}
\sum_{k=0}^{M} E\left[\Delta V\left(x_{k}, r_{k}\right)\right] & =E\left[V\left(x_{M+1}, r_{M+1}\right)\right]-V\left(x_{0}, r_{0}\right) \\
& \leq-\rho \sum_{k=0}^{M} E\left(\left\|x_{k}\right\|^{2}\right) .
\end{aligned}
$$

Hence,

$$
\begin{aligned}
\sum_{k=0}^{M} E\left(\left\|x_{k}\right\|^{2}\right) & \leq \frac{1}{\rho}\left[V\left(x_{0}, r_{0}\right)-E\left[V\left(x_{M+1}, r_{M+1}\right)\right]\right] \\
& \leq \frac{1}{\rho} V\left(x_{0}, r_{0}\right)
\end{aligned}
$$

reducing to

$$
\begin{aligned}
\sum_{k=0}^{\infty} E\left(\left\|x_{k}\right\|^{2}\right) & =\lim _{M \rightarrow \infty} \sum_{k=0}^{M} E\left(\left\|x_{k}\right\|^{2}\right) \leq \frac{1}{\rho} V\left(x_{0}, r_{0}\right) \\
& <\infty .
\end{aligned}
$$

Therefore, system (1) is stochastically stable from Definition 2. The proof is completed.

Remark 5. Theorem 4 develops a sufficient condition for SS of system (1) when transition probability matrix is arbitrarily valued in a polytope with given vertices. For finite Markov jump switching systems, the stochastic stability is equivalent to asymptotic mean square stability (AMSS) [1]. So Theorem 4 is also a sufficient condition for AMSS.

Remark 6. A convex parameter space method was used in control design of uncertain linear system without jumping. State feedback conditions can be easily derived by exploiting the change of matrix variable due to Geromel and coworkers [31].

It is hard to verify the conditions in Theorem 4 because there are infinite matrix inequalities. Hence it has more theoretical significance than its practical significance. To be solvable, we introduce a set of slack matrices and convert the infinite matrix inequalities into finite LMIs in the following theorem. 
Theorem 7. The following two statements are equivalent:

(1) There exist a set of matrices $P_{i}^{l}>0, \forall i \in S$, and $l=$ $1,2, \ldots, m$, such that (7) holds for every $\alpha_{l}(k)$ and $\beta_{n}(k), l, n=$ $1,2, \ldots, m$, satisfying $0 \leq \alpha_{l}(k), \beta_{n}(k) \leq 1, \sum_{l=1}^{m} \alpha_{l}(k)=1$, and $\sum_{n=1}^{m} \beta_{n}(k)=1$.

(2) There exist a set of matrices $Q_{i}^{l}>0$ and matrices $G_{i}^{l}$, such that

$$
\left[\begin{array}{ccc}
G_{i}^{l}+\left(G_{i}^{l}\right)^{\mathrm{T}}-Q_{i}^{l} & \left(G_{i}^{l}\right)^{\mathrm{T}} A_{i}^{\mathrm{T}} \Gamma_{i}^{l} & \left(G_{i}^{l}\right)^{\mathrm{T}} C_{i}^{\mathrm{T}} \Gamma_{i}^{l} \\
* & \bar{Q}^{n} & 0 \\
* & * & \bar{Q}^{n}
\end{array}\right]>0,
$$

$$
\forall i \in S, l, n=1,2, \ldots, m \text {, }
$$

where $\Gamma_{i}^{l}=\left[\sqrt{\pi_{i 1}^{l}} \mathbf{I}, \ldots, \sqrt{\pi_{i s}^{l}} \mathbf{I}\right]$ and $\overline{\mathbf{Q}}^{n}=\operatorname{diag}\left(Q_{1}^{n}, \ldots, Q_{s}^{n}\right)$.

The proof is similar to Proposition 2 in [12], so it is omitted.

From Theorem 7, if $A_{i}, C_{i}$ are replaced with $A_{i}+B_{i} K_{i}, C_{i}$ $+D_{i} K_{i}$, respectively, it is easy to get the following stabilization condition and controller design method.

Corollary 8. If there exist a set of matrices $Q_{i}^{l}>0$ and matrices $G_{i}, R_{i}$ such that the LMIs

$$
\left[\begin{array}{ccc}
G_{i}+G_{i}^{\mathrm{T}}-Q_{i}^{l} & \left(G_{i}^{\mathrm{T}} A_{i}^{\mathrm{T}}+R_{i}^{\mathrm{T}} B_{i}^{\mathrm{T}}\right) \Gamma_{i}^{l} & \left(G_{i}^{\mathrm{T}} C_{i}^{\mathrm{T}}+R_{i}^{\mathrm{T}} D_{i}^{\mathrm{T}}\right) \Gamma_{i}^{l} \\
* & \overline{\mathrm{Q}}^{n} & 0 \\
* & * & \overline{\mathrm{Q}}^{n}
\end{array}\right]
$$

$>0, \quad \forall i \in S, l, n=1,2, \ldots, m$,

hold, then system (2) is stochastically stabilized by $u_{k}=$ $K\left(r_{k}\right) x_{k}$ with the feedback gains $K_{i}=R_{i} G_{i}^{-1}$.

\section{Finite-Time Stability and Control}

In this section, we will consider the finite-time stability. Firstly a theoretical criterion is given.

Theorem 9. If there exist a scalar $\gamma \geq 1$ and matrices $P_{i}^{l}>0$, $\forall i \in S$, such that

$$
\begin{aligned}
& A_{i}^{\mathrm{T}}\left(\sum_{j=1}^{s} \sum_{l=1}^{m} \sum_{n=1}^{m} \alpha_{l}(k) \beta_{n}(k) \pi_{i j}^{l} P_{j}^{n}\right) A_{i} \\
& \quad+C_{i}^{\mathrm{T}}\left(\sum_{j=1}^{s} \sum_{l=1}^{m} \sum_{n=1}^{m} \alpha_{l}(k) \beta_{n}(k) \pi_{i j}^{l} P_{j}^{n}\right) C_{i} \\
& \quad-\gamma \sum_{l=1}^{m} \alpha_{l}(k) P_{i}^{l}<0, \\
& c_{1} \gamma^{T} \operatorname{Cond}\left(\bar{P}_{i}\right)<c_{2}, \\
& \bar{P}_{i}=\sum_{l=1}^{m} \alpha_{l}(k) R^{-1 / 2} P_{i}^{l} R^{-1 / 2},
\end{aligned}
$$

where $0 \leq \alpha_{l}(k), \beta_{n}(k) \leq 1, \sum_{l=1}^{m} \alpha_{l}(k)=1$, and $\sum_{n=1}^{m} \beta_{n}(k)=$ 1 , then system (1) is finite-time stable with respect to $\left(c_{1}, c_{2}, R\right.$, T).

Proof. Let $V\left(x_{k}, r_{k}=i\right)=x_{k}^{\mathrm{T}}\left(\sum_{l=1}^{m} \alpha_{l}(k) P_{i}^{l}\right) x_{k}$. Applying the same procedure as the proof of Theorem 4 , we can get

$$
\begin{aligned}
E & {\left[V\left(x_{k+1}, r_{k+1}=j\right)\right] } \\
& =x_{k}^{\mathrm{T}}\left[A_{i}^{\mathrm{T}}\left(\sum_{j=1}^{s} \sum_{l=1}^{m} \sum_{n=1}^{m} \alpha_{l}(k) \beta_{n}(k) \pi_{i j}^{l} P_{i}^{l}\right) A_{i}\right. \\
& \left.+C_{i}^{\mathrm{T}}\left(\sum_{j=1}^{s} \sum_{l=1}^{m} \sum_{n=1}^{m} \alpha_{l}(k) \beta_{n}(k) \pi_{i j}^{l} P_{i}^{l}\right) C_{i}\right] x_{k} .
\end{aligned}
$$

It follows from (15) that $E\left[V\left(x_{k+1}, r_{k+1}\right)\right]<\gamma E\left[V\left(x_{k}, r_{k}\right)\right]$; that is,

$$
E\left[V\left(x_{k}, r_{k}\right)\right]<\gamma^{k} E\left[V\left(x_{0}, r_{0}\right)\right] \leq \gamma^{T} E\left[V\left(x_{0}, r_{0}\right)\right] .
$$

While

$$
\begin{aligned}
E\left[V\left(x_{0}, r_{0}\right)\right] & =x_{0}^{\mathrm{T}}\left(\sum_{l=1}^{m} \alpha_{l}(k) P_{i}^{l}\right) x_{0} \\
& =x_{0}^{\mathrm{T}} R^{1 / 2} \bar{P}_{i} R^{1 / 2} x_{0} \leq \lambda_{\max }\left(\bar{P}_{i}\right) x_{0}^{\mathrm{T}} R x_{0} \\
& \leq \lambda_{\max }\left(\bar{P}_{i}\right) c_{1}, \\
E\left[V\left(x_{k}, r_{k}\right)\right] & =E\left[x_{k}^{\mathrm{T}}\left(\sum_{l=1}^{m} \alpha_{l}(k) P_{i}^{l}\right) x_{k}\right] \\
& =E\left[x_{k}^{\mathrm{T}} R^{1 / 2} \bar{P}_{i} R^{1 / 2} x_{k}\right] \\
& \geq \lambda_{\min }\left(\bar{P}_{i}\right) E\left(x_{k}^{\mathrm{T}} R x_{k}\right),
\end{aligned}
$$

so

$$
\begin{aligned}
E\left(x_{k}^{\mathrm{T}} R x_{k}\right) & \leq \frac{E\left[V\left(x_{k}, r_{k}\right)\right]}{\lambda_{\min }\left(\bar{P}_{i}\right)} \leq \frac{c_{1} \gamma^{T} \lambda_{\text {max }}\left(\bar{P}_{i}\right)}{\lambda_{\text {min }}\left(\bar{P}_{i}\right)} \\
& =c_{1} \gamma^{T} \operatorname{Cond}\left(\bar{P}_{i}\right)<c_{2} .
\end{aligned}
$$

Hence, from Definition 3, system (1) is finite-time stable with respect to $\left(c_{1}, c_{2}, R, T\right)$. The proof is completed.

Next, we convert the infinite matrix inequalities (15) and (16) into finite matrix inequalities by use of slack matrices and properties of the condition number and design a state feedback controller to make system (2) finite-time stabilizable.

Theorem 10. (1) If there exist a scalar $\gamma \geq 1$, matrices $Q_{i}^{l}>0$, and matrices $G_{i}^{l}$, such that 


$$
\left[\begin{array}{ccc}
\gamma\left[G_{i}^{l}+\left(G_{i}^{l}\right)^{\mathrm{T}}-Q_{i}^{l}\right] & \left(G_{i}^{l}\right)^{\mathrm{T}} A_{i}^{\mathrm{T}} \Gamma_{i}^{l} & \left(G_{i}^{l}\right)^{\mathrm{T}} C_{i}^{\mathrm{T}} \Gamma_{i}^{l} \\
* & \overline{\mathrm{Q}}^{n} & 0 \\
* & * & \overline{\mathrm{Q}}^{n}
\end{array}\right]
$$

$>0, \quad \forall i \in S, l, n=1,2, \ldots, m$,

$c_{1} \gamma^{T} \max _{l=1, \ldots, m}\left\{\operatorname{Cond}\left(R^{1 / 2} Q_{i}^{l} R^{1 / 2}\right)\right\}<c_{2}, \quad \forall i \in S$,

where $\Gamma_{i}^{l}=\left[\sqrt{\pi_{i 1}^{l}} \mathbf{I}, \ldots, \sqrt{\pi_{i s}^{l}} \mathbf{I}\right]$ and $\bar{Q}^{n}=\operatorname{diag}\left(Q_{1}^{n}, \ldots, Q_{s}^{n}\right)$, then system (1) is finite-time stable.

(2) If there exist a scalar $\gamma \geq 1$, matrices $Q_{i}^{l}>0$, and matrices $G_{i}, R_{i}$ such that

$$
\left[\begin{array}{ccc}
\gamma\left[G_{i}+G_{i}^{\mathbf{T}}-Q_{i}^{l}\right] & \left(G_{i}^{\mathbf{T}} A_{i}^{\mathbf{T}}+R_{i}^{\mathrm{T}} B_{i}^{\mathrm{T}}\right) \Gamma_{i}^{l} & \left(G_{i}^{\mathbf{T}} C_{i}^{\mathbf{T}}+R_{i}^{\mathbf{T}} D_{i}^{\mathrm{T}}\right) \Gamma_{i}^{l} \\
* & \bar{Q}^{n} & 0 \\
* & * & \bar{Q}^{n}
\end{array}\right]
$$

$>0, \quad \forall i \in S, l, n=1,2, \ldots, m$,

and (22) hold, then system (2) is finite-time stabilized by $u_{k}=$ $K\left(r_{k}\right) x_{k}$ with the feedback gains $K_{i}=R_{i} G_{i}^{-1}$.

Proof. (1) The proof can be divided into two parts. Firstly, we prove that (15) holds if (21) holds. We adopt the method used in Proposition 2 of [12].
From (21), $G_{i}^{l}+\left(G_{i}^{l}\right)^{\mathrm{T}}-Q_{i}^{l}>0$. As $Q_{i}^{l}$ is positive definite, $G_{i}^{l}+\left(G_{i}^{l}\right)^{\mathrm{T}}$ must be positive definite. While $\left|(1 / 2)\left(G_{i}^{l}+\left(G_{i}^{l}\right)^{\mathbf{T}}\right)\right| \leq$ $\left|G_{i}^{l}\right|$, it follows that $G_{i}^{l}$ is invertible. On the other hand,

$$
\begin{aligned}
\gamma & {\left[\left(Q_{i}^{l}-G_{i}^{l}\right)^{\mathrm{T}}\left(Q_{i}^{l}\right)^{-1}\left(Q_{i}^{l}-G_{i}^{l}\right)\right] } \\
& =\gamma\left[\left(Q_{i}^{l}\right)^{-1 / 2}\left(Q_{i}^{l}-G_{i}^{l}\right)\right]^{\mathrm{T}}\left[\left(Q_{i}^{l}\right)^{-1 / 2}\left(Q_{i}^{l}-G_{i}^{l}\right)\right]
\end{aligned}
$$

$\geq 0$,

which means that $\gamma\left(G_{i}^{l}\right)^{\mathrm{T}}\left(Q_{i}^{l}\right)^{-1} G_{i}^{l} \geq \gamma\left[G_{i}^{l}+\left(G_{i}^{l}\right)^{\mathbf{T}}-Q_{i}^{l}\right]$. Then,

$$
\begin{aligned}
& {\left[\begin{array}{ccc}
\gamma\left(G_{i}^{l}\right)^{\mathrm{T}}\left(Q_{i}^{l}\right)^{-1} G_{i}^{l} & \left(G_{i}^{l}\right)^{\mathrm{T}} A_{i}^{\mathrm{T}} \Gamma_{i}^{l} & \left(G_{i}^{l}\right)^{\mathrm{T}} C_{i}^{\mathrm{T}} \Gamma_{i}^{l} \\
* & \overline{\mathrm{Q}}^{n} & 0 \\
* & * & \overline{\mathrm{Q}}^{n}
\end{array}\right]} \\
& \geq\left[\begin{array}{ccc}
\gamma\left[G_{i}^{l}+\left(G_{i}^{l}\right)^{\mathrm{T}}-Q_{i}^{l}\right] & \left(G_{i}^{l}\right)^{\mathrm{T}} A_{i}^{\mathrm{T}} \Gamma_{i}^{l}\left(G_{i}^{l}\right)^{\mathrm{T}} C_{i}^{\mathrm{T}} \Gamma_{i}^{l} \\
* & \bar{Q}^{n} & 0 \\
* & * & \bar{Q}^{n}
\end{array}\right]
\end{aligned}
$$

$>0$,

which is equivalent to

$$
\left[\begin{array}{ccc}
\left(G_{i}^{l}\right)^{\mathrm{T}} & 0 & 0 \\
* & \overline{\mathrm{Q}}^{n} & 0 \\
* & * & \overline{\mathrm{Q}}^{n}
\end{array}\right]\left[\begin{array}{ccc}
\gamma\left(Q_{i}^{l}\right)^{-1} & A_{i}^{\mathrm{T}} \Gamma_{i}^{l}\left(\overline{\mathrm{Q}}^{n}\right)^{-1} & C_{i}^{\mathrm{T}} \Gamma_{i}^{l}\left(\overline{\mathrm{Q}}^{n}\right)^{-1} \\
* & \left(\overline{\mathrm{Q}}^{n}\right)^{-1} & 0 \\
* & * & \left(\overline{\mathrm{Q}}^{n}\right)^{-1}
\end{array}\right]\left[\begin{array}{ccc}
G_{i}^{l} & 0 & 0 \\
* & \overline{\mathrm{Q}}^{n} & 0 \\
* & * & \overline{\mathrm{Q}}^{n}
\end{array}\right]>0 .
$$

Because of the invertibility of $G_{i}^{l}$ and $\bar{Q}^{n}$, we have

$$
\left[\begin{array}{ccc}
\gamma\left(Q_{i}^{l}\right)^{-1} & A_{i}^{\mathrm{T}} \Gamma_{i}^{l}\left(\bar{Q}^{n}\right)^{-1} & C_{i}^{\mathrm{T}} \Gamma_{i}^{l}\left(\overline{\mathrm{Q}}^{n}\right)^{-1} \\
* & \left(\overline{\mathrm{Q}}^{n}\right)^{-1} & 0 \\
* & * & \left(\overline{\mathrm{Q}}^{n}\right)^{-1}
\end{array}\right]>0 .
$$

Let $P_{i}^{l}=\left(Q_{i}^{l}\right)^{-1}$; then (27) can be rewritten as

$$
\left[\begin{array}{ccc}
\gamma P_{i}^{l} & A_{i}^{\mathrm{T}} \Gamma_{i}^{l} \bar{P}^{n} & C_{i}^{\mathrm{T}} \Gamma_{i}^{l} \bar{P}^{n} \\
* & \bar{P}^{n} & 0 \\
* & * & \bar{P}^{n}
\end{array}\right]>0,
$$

where $\bar{P}^{n}=\operatorname{diag}\left(P_{1}^{n}, P_{2}^{n}, \ldots, P_{s}^{n}\right)$. By Schur complement, (28) is equivalent to

$$
\gamma P_{i}^{l}-A_{i}^{\mathrm{T}}\left(\sum_{j=1}^{s} \pi_{i j}^{l} P_{j}^{n}\right) A_{i}-C_{i}^{\mathrm{T}}\left(\sum_{j=1}^{s} \pi_{i j}^{l} P_{j}^{n}\right) C_{i}>0 .
$$

Obviously, (15) holds after multiplying (29) by the corresponding coefficients and adding them.

Next, we prove that (16) holds if (22) is true. Before proving this, we need to recall two properties of the condition number:

(a) $\operatorname{Cond}(c A)=\operatorname{Cond}(A)=\operatorname{Cond}\left(A^{-1}\right), c>0$;

(b) $\operatorname{Cond}\left(A_{1}+A_{2}\right) \leq \max \left\{\operatorname{Cond}\left(A_{1}\right)\right.$, $\left.\operatorname{Cond}\left(A_{2}\right)\right\}$.

It follows from (a) and (b) that

$$
\begin{array}{r}
\text { Cond }\left(\alpha_{1} A_{1}+\alpha_{2} A_{2}+\cdots+\alpha_{n} A_{n}\right) \\
\leq \max _{i=1,2, \ldots, n}\left\{\operatorname{Cond}\left(A_{i}\right)\right\}, \quad \alpha_{i}>0 .
\end{array}
$$

When (22) holds and $P_{i}^{l}=\left(Q_{i}^{l}\right)^{-1}$, one has

$$
\begin{aligned}
\bar{P}_{i} & =\sum_{l=1}^{m} \alpha_{l}(k) R^{-1 / 2} P_{i}^{l} R^{-1 / 2} \\
& =\sum_{l=1}^{m} \alpha_{l}(k) R^{-1 / 2}\left(Q_{i}^{l}\right)^{-1} R^{-1 / 2},
\end{aligned}
$$




$$
\begin{aligned}
& c_{1} \gamma^{T} \operatorname{Cond}\left(\bar{P}_{i}\right) \\
& \quad \leq c_{1} \gamma^{T} \max _{l=1, \ldots, m}\left\{\operatorname{Cond}\left(R^{-1 / 2}\left(Q_{i}^{l}\right)^{-1} R^{-1 / 2}\right)\right\} \\
& \quad=c_{1} \gamma^{T} \max _{l=1, \ldots, m}\left\{\operatorname{Cond}\left(R^{1 / 2} Q_{i}^{l} R^{1 / 2}\right)\right\}<c_{2} .
\end{aligned}
$$

(2) This can be easily proved by the same procedure as (1).

Remark 11. We should point out that unlike the equivalence of (7) and (13), (21) is just a sufficient condition of (15) due to the existence of $\gamma$.

Remark 12. In fact, (22) can be guaranteed by the following LMIs:

$$
c_{1} \gamma^{T} \mathbf{I}<R^{1 / 2} Q_{i}^{l} R^{1 / 2}<c_{2} \mathbf{I}, \quad l=1,2, \ldots, m .
$$

So we can obtain a set of feasible feedback controls by solving (23) and (32) or by solving (23) and checking if they satisfy (22).

\section{Numerical Examples}

In this section, two illustrative examples are presented to show the effectiveness of the proposed main results.

Example 1. Consider system (2) with coefficient matrices as follows:

$$
\begin{aligned}
& A_{1}=\left[\begin{array}{cc}
0.22 & 1.52 \\
-1.10 & 1.37
\end{array}\right] \text {, } \\
& A_{2}=\left[\begin{array}{cc}
0.17 & -0.64 \\
0.73 & 1.05
\end{array}\right] \text {, } \\
& A_{3}=\left[\begin{array}{ll}
0.47 & 0.11 \\
0.14 & 0.30
\end{array}\right] \text {, } \\
& B_{1}=\left[\begin{array}{cc}
0.73 & 1.13 \\
-0.47 & 1.37
\end{array}\right] \text {, } \\
& B_{2}=\left[\begin{array}{cc}
-0.02 & 1.18 \\
0.37 & -0.46
\end{array}\right] \text {, } \\
& B_{3}=\left[\begin{array}{cc}
-0.47 & 0.11 \\
0.14 & -1.30
\end{array}\right] \text {, } \\
& C_{1}=\left[\begin{array}{cc}
1.10 & -0.46 \\
1.86 & 1.23
\end{array}\right] \text {, } \\
& C_{2}=\left[\begin{array}{cc}
1.45 & -0.10 \\
-0.49 & 0.17
\end{array}\right] \text {, } \\
& C_{3}=\left[\begin{array}{cc}
-1.01 & 0.20 \\
0.04 & 1.20
\end{array}\right] \text {, }
\end{aligned}
$$

$$
\begin{aligned}
& D_{1}=\left[\begin{array}{cc}
1.05 & -0.24 \\
0.89 & 1.01
\end{array}\right], \\
& D_{2}=\left[\begin{array}{cc}
1.05 & -0.11 \\
0.55 & 0.41
\end{array}\right], \\
& D_{3}=\left[\begin{array}{cc}
-0.51 & 0.25 \\
1.23 & 0.21
\end{array}\right] .
\end{aligned}
$$

The initial state is given as $x_{0}=(-0.5,0.5)^{\mathrm{T}}$. The vertices of the polytope transition probability matrices are given as follows:

$$
\begin{aligned}
\Gamma^{1} & =\left[\begin{array}{ccc}
0.3 & 0.5 & 0.2 \\
0.5 & 0.5 & 0 \\
0.1 & 0.4 & 0.5
\end{array}\right], \\
\Gamma^{2} & =\left[\begin{array}{ccc}
0 & 1 & 0 \\
0.1 & 0.1 & 0.8 \\
0.3 & 0.4 & 0.3
\end{array}\right], \\
\Gamma^{3} & =\left[\begin{array}{lll}
0.4 & 0.2 & 0.4 \\
0.2 & 0.1 & 0.7 \\
0.1 & 0.3 & 0.6
\end{array}\right] .
\end{aligned}
$$

By solving LMIs (14), it can be concluded that system (2) is stochastically stabilized via feedback gains

$$
\begin{aligned}
& K_{1}=\left[\begin{array}{cc}
-1.1058 & -0.0365 \\
0.1262 & -1.2281
\end{array}\right], \\
& K_{2}=\left[\begin{array}{cc}
-1.2668 & -0.0051 \\
0.0747 & 0.6577
\end{array}\right], \\
& K_{3}=\left[\begin{array}{cc}
-0.3565 & -0.4793 \\
0.0552 & -0.0928
\end{array}\right] .
\end{aligned}
$$

The system modes and the state trajectories of the openloop and closed-loop systems are, respectively, shown in Figures 1-3.

Example 2. Consider system (2) with coefficient matrices as follows:

$$
\begin{gathered}
A_{1}=\left[\begin{array}{cc}
0.22 & -0.78 \\
0.56 & 0.01
\end{array}\right], \\
A_{2}=\left[\begin{array}{cc}
0.27 & -0.85 \\
0.23 & 0.58
\end{array}\right], \\
B_{1}=\left[\begin{array}{c}
0.07 \\
-0.93
\end{array}\right], \\
B_{2}=\left[\begin{array}{c}
0.91 \\
-0.18
\end{array}\right],
\end{gathered}
$$




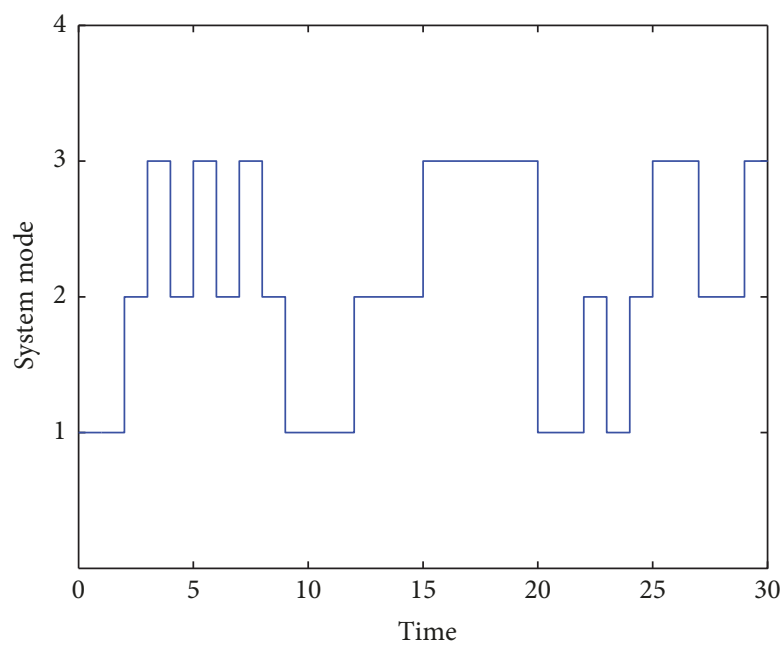

FIgURE 1: The system modes for Example 1.
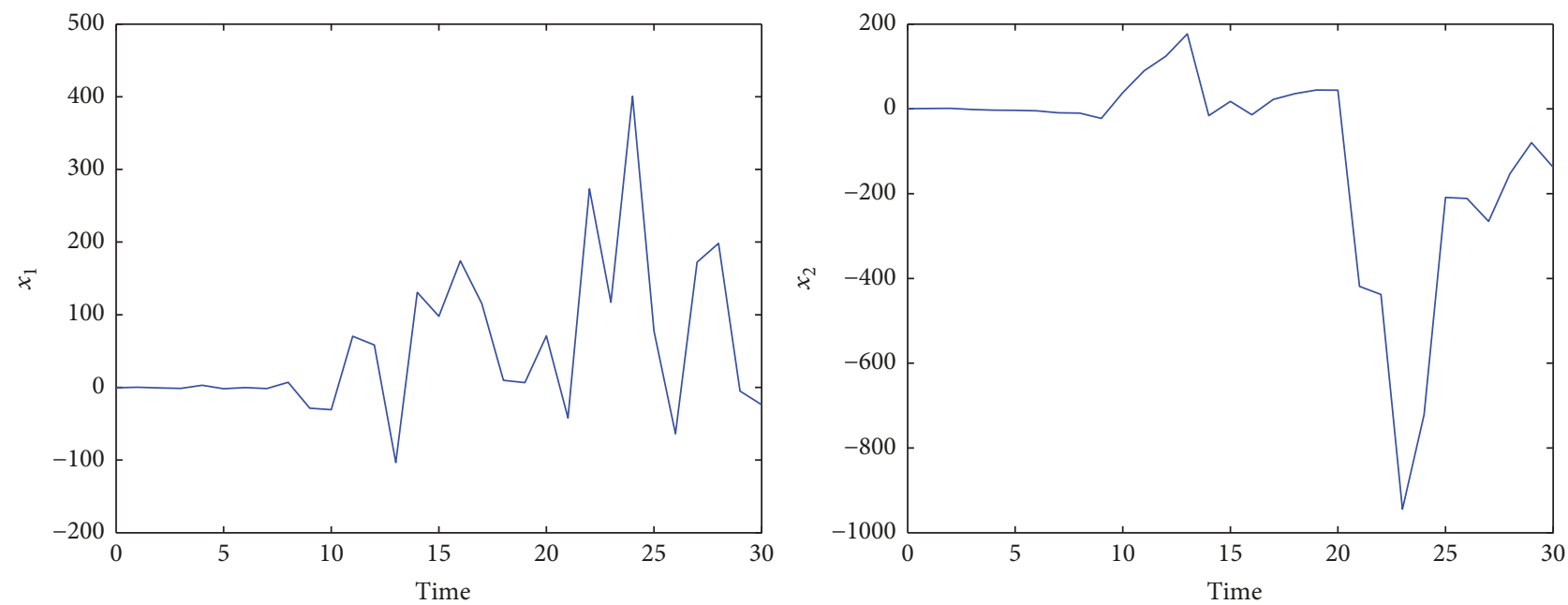

FIgURE 2: The states of open-loop system for Example 1.
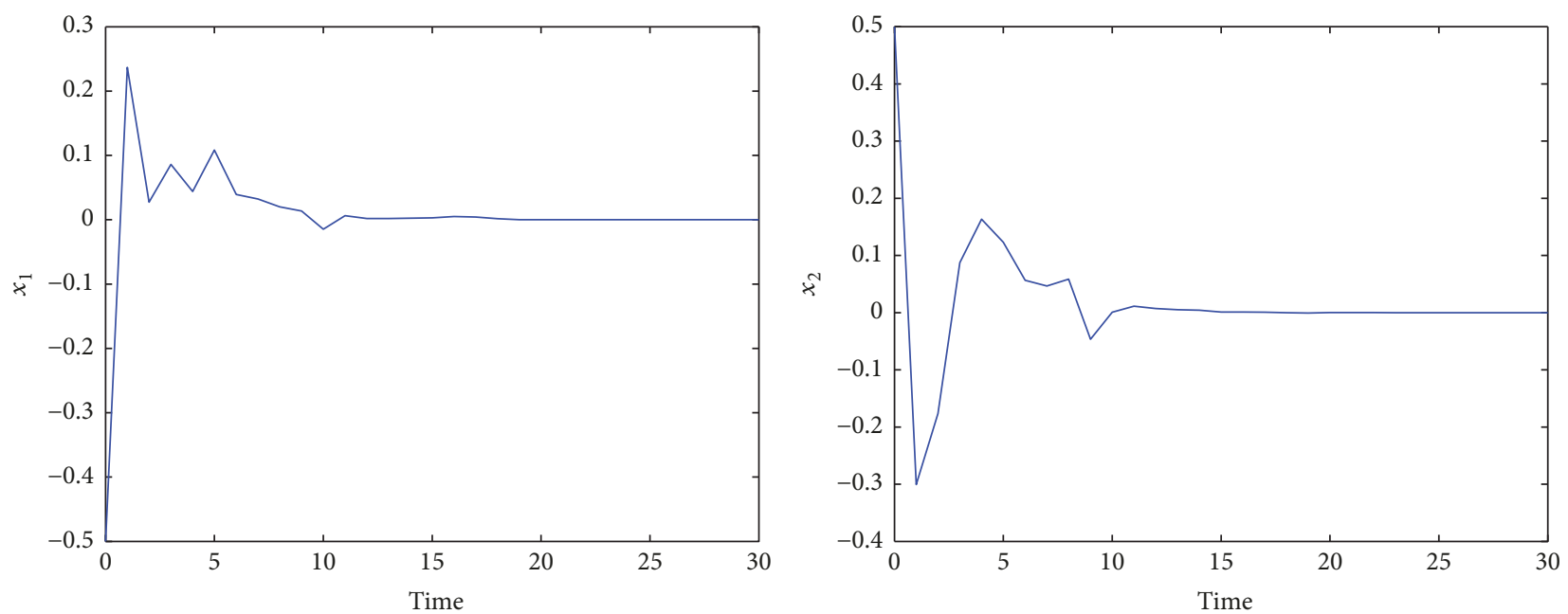

Figure 3: The states of closed-loop system for Example 1. 


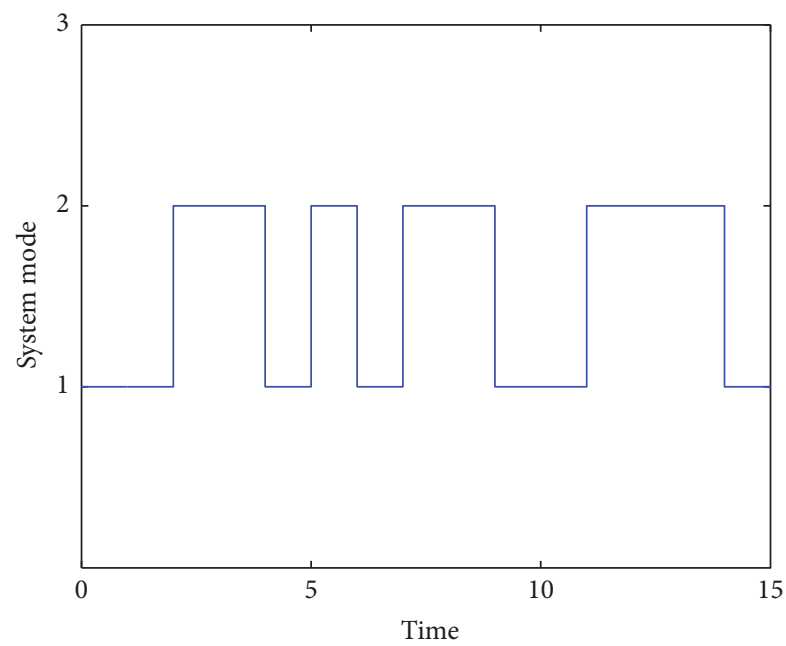

FIGURE 4: The system modes for Example 2.

$$
\begin{gathered}
C_{1}=\left[\begin{array}{cc}
0.06 & 0.45 \\
-0.44 & -0.52
\end{array}\right], \\
C_{2}=\left[\begin{array}{ll}
-1.07 & 0.06 \\
-0.89 & 0.46
\end{array}\right], \\
D_{1}=\left[\begin{array}{c}
-0.04 \\
0.27
\end{array}\right], \\
D_{2}=\left[\begin{array}{c}
0.15 \\
-0.11
\end{array}\right] .
\end{gathered}
$$

The initial state is given as $x_{0}=(-2,1)^{\mathrm{T}}$ and the vertices of the polytope transition probability matrices are given as follows:

$$
\begin{aligned}
\Gamma^{1} & =\left[\begin{array}{ll}
0.1 & 0.9 \\
0.5 & 0.5
\end{array}\right], \\
\Gamma^{2} & =\left[\begin{array}{cc}
0.4 & 0.6 \\
1 & 0
\end{array}\right], \\
\Gamma^{3} & =\left[\begin{array}{ll}
0.2 & 0.8 \\
0.3 & 0.7
\end{array}\right] .
\end{aligned}
$$

Given $c_{1}=5, c_{2}=70, R=\mathbf{I}$, and $T=15$ and choosing $\gamma=1.05$, by solving LMIs (23) and checking (22), it can be concluded that system (2) is finite-time stabilized via feedback gains

$$
\begin{aligned}
& K_{1}=\left[\begin{array}{ll}
0.5537 & 0.6242
\end{array}\right], \\
& K_{2}=\left[\begin{array}{ll}
-0.1028 & 1.1531
\end{array}\right] .
\end{aligned}
$$

The system modes and the state trajectories of the openloop and closed-loop systems are, respectively, shown in Figures 4-6.

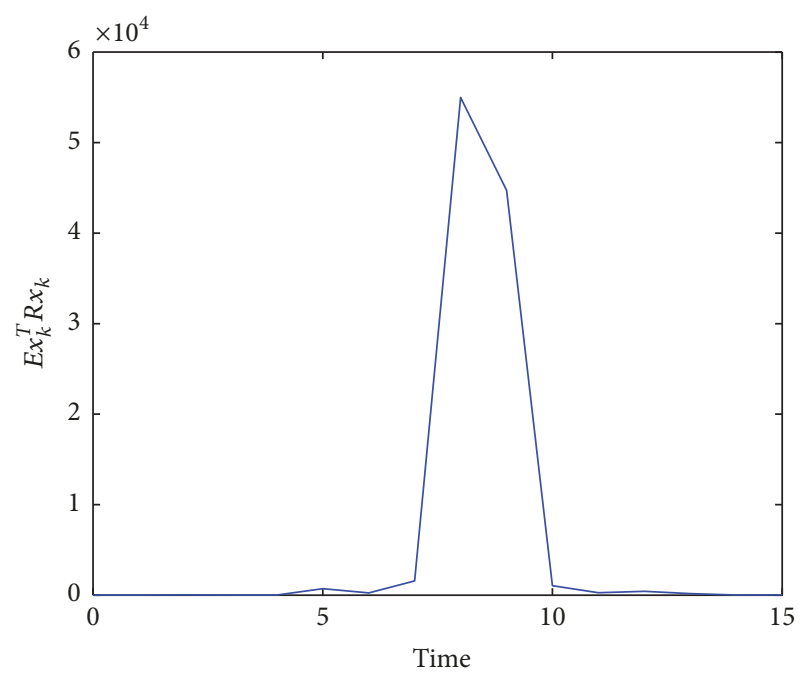

Figure 5: $E\left(x_{k}^{\mathrm{T}} R x_{k}\right)$ of the open-loop system for Example 2.

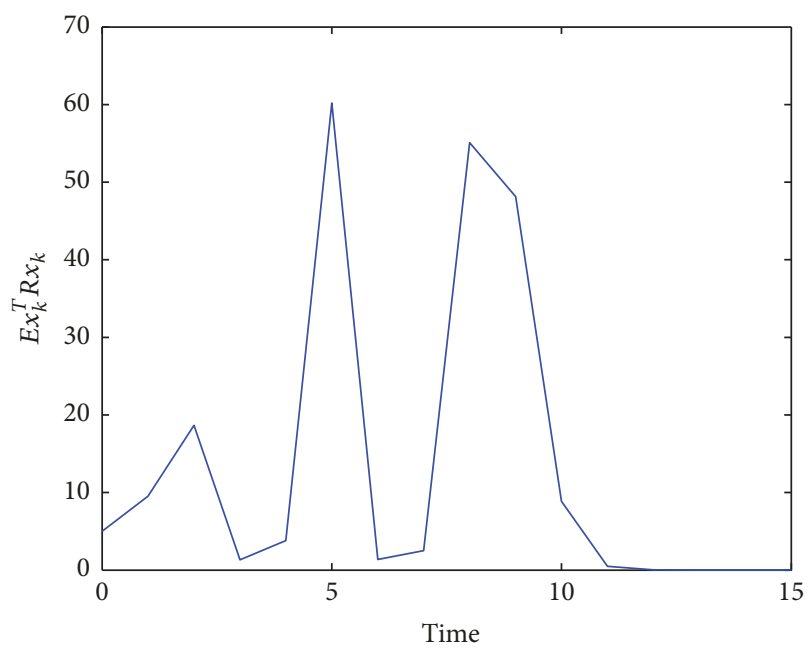

FIGURE 6: $E\left(x_{k}^{\mathrm{T}} R x_{k}\right)$ of the closed-loop system for Example 2.

\section{Conclusions}

In this paper, we have investigated the stochastic stability and finite-time stability of a kind of discrete-time nonhomogeneous Markov jump systems with multiplicative noises and polytopic transition probability matrices. Some sufficient conditions for stability are proposed by parameter-dependent Lyapunov function and stabilization controllers are designed by using LMI toolbox. The simulation results show the effectiveness of the developed techniques. This research will motivate us to study the continuous-time version in future.

\section{Conflicts of Interest}

The authors declare that they have no conflicts of interest.

\section{Acknowledgments}

This work was supported by the National Natural Science Foundation of China $(61473160,61503224)$, the Special Funds 
for Postdoctoral Innovative Projects of Shandong Province (201403009), the Research Award Funds for Outstanding Young Scientists of Shandong Province (BS2014SF005), and SDUST Research Fund (2015TDJH105).

\section{References}

[1] O. L. V. Costa, M. D. Fragoso, and R. P. Marques, Discrete-Time Markov Jump Linear Systems, Springer, London, UK, 2005.

[2] O. L. V. Costa, M. D. Fragoso, and M. G. Todorov, ContinuousTime Markov Jump Linear Systems, Springer, Berlin, Germany, 2013.

[3] L. Zhang, T. Yang, P. Shi, and Y. Zhu, Analysis and Design of Markov Jump Systems with Complex Transition Probabilities, Springer, Switzerland, 2016.

[4] X. Mao and C. Yuan, Stochastic Differential Equations with Markovian Switching, Imperial College Press, London, UK, 2006.

[5] Y. Fang, Stability analysis of linear control systems with uncertain parameters, Case Western Reserve University, Cleveland, $\mathrm{OH}$, USA, 1994.

[6] L.X. Zhang, " $H_{\infty}$ estimation for discrete-time piecewise homogeneous Markov jump linear systems," Automatica, vol. 45, no. 11, pp. 2570-2576, 2009.

[7] M. Faraji-Niri, M. R. Jahed-Motlagh, and M. BarkhordariYazdi, "Robust stabilization of uncertain non-homogeneous Markov jump linear systems," in Proceedings of the 3rd International Conference on Control, Instrumentation, and Automation, pp. 42-46, Tehran, Iran, December 2013.

[8] Z.-G. Wu, J. H. Park, H. Su, and J. Chu, "Stochastic stability analysis of piecewise homogeneous Markovian jump neural networks with mixed time-delays," Journal of The Franklin Institute, vol. 349, no. 6, pp. 2136-2150, 2012.

[9] Z.-G. Wu, J. H. Park, H. Su, and J. Chu, "Passivity analysis of Markov jump neural networks with mixed time-delays and piecewise-constant transition rates," Nonlinear Analysis: Real World Applications, vol. 13, no. 5, pp. 2423-2431, 2012.

[10] Z.-X. Li, J. H. Park, and Z.-G. Wu, "Synchronization of complex networks with nonhomogeneous Markov jump topology," Nonlinear Dynamics, vol. 74, no. 1-2, pp. 65-75, 2013.

[11] Z.-G. Wu, J. H. Park, H. Su, and J. Chu, "Stochastic stability analysis for discrete-time singular Markov jump systems with timevarying delay and piecewise-constant transition probabilities," Journal of The Franklin Institute, vol. 349, no. 9, pp. 2889-2902, 2012.

[12] S. Aberkane, "Stochastic stabilization of a class of nonhomogeneous Markovian jump linear systems," Systems \& Control Letters, vol. 60, no. 3, pp. 156-160, 2011.

[13] Y. Yin, P. Shi, F. Liu, and K. L. Teo, "Filtering for discrete-time nonhomogeneous Markov jump systems with uncertainties," Information Sciences, vol. 259, pp. 118-127, 2014.

[14] Y. Yin, P. Shi, F. Liu, and K. L. Teo, "Observer-based $H_{\infty}$ control on nonhomogeneous discrete-time markov jump systems," Journal of Dynamic Systems, Measurement, and Control, vol. 135, no. 4, Article ID 041016, 2013.

[15] P. Shi, Y. Yin, F. Liu, and J. Zhang, "Robust control on saturated Markov jump systems with missing information," Information Sciences, vol. 265, pp. 123-138, 2014.

[16] Y. Zhang, Y. Ou, Y. Zhou, X. Wu, and W. Sheng, "Observerbased $l_{2}-l_{\infty}$ control for discrete-time nonhomogeneous Markov jump Lur'e systems with sensor saturations," Neurocomputing, vol. 162, pp. 141-149, 2015.

[17] Y. Long and G.-H. Yang, "Fault detection for a class of nonhomogeneous Markov jump systems based on delta operator approach," Proceedings of the Institution of Mechanical Engineers, Part I: Journal of Systems and Control Engineering, vol. 227, no. 1, pp. 129-141, 2013.

[18] Y. Yin, P. Shi, F. Liu, and C. C. Lim, "Robust control for nonhomogeneous Markov jump processes: an application to DC motor device," Journal of The Franklin Institute, vol. 351, no. 6, pp. 3322-3338, 2014.

[19] Y. Ding and H. Liu, "Stability analysis of continuous-time Markovian jump time-delay systems with time-varying transition rates," Journal of The Franklin Institute, vol. 353, no. 11, pp. 2418-2430, 2016.

[20] S. Aberkane, "Bounded real lemma for nonhomogeneous Markovian jump linear systems," Institute of Electrical and Electronics Engineers Transactions on Automatic Control, vol. 58, no. 3, pp. 797-801, 2013.

[21] T. Hou, H. Ma, and W. Zhang, "Spectral tests for observability and detectability of periodic Markov jump systems with nonhomogeneous Markov chain," Automatica, vol. 63, pp. 175-181, 2016.

[22] L. Qian and Z. Gajic, "Variance minimization stochastic power control in CDMA systems," in Proceedings of the IEEE International Conference on Communications, vol. 3, pp. 1763-1767, New York, NY, USA, 2002.

[23] F. Amato and M. Ariola, "Finite-time control of discrete-time linear systems," Institute of Electrical and Electronics Engineers Transactions on Automatic Control, vol. 50, no. 5, pp. 724-729, 2005.

[24] F. Amato, R. Ambrosino, M. Ariola, C. Cosentino, and G. De Tommasi, Finite-time stability and control, Springer, London, UK, 2014.

[25] F. Amato, M. Ariola, and C. Cosentino, "Robust finite-time stabilisation of uncertain linear systems," International Journal of Control, vol. 84, no. 12, pp. 2117-2127, 2011.

[26] F. Tan, B. Zhou, and G.-R. Duan, "Finite-time stabilization of linear time-varying systems by piecewise constant feedback," Automatica, vol. 68, pp. 277-285, 2016.

[27] X. Luan, F. Liu, and P. Shi, "Observer-based finite-time stabilization for extended Markov jump systems," Asian Journal of Control, vol. 13, no. 6, pp. 925-935, 2011.

[28] Z. Yan, W. Zhang, and G. Zhang, "Finite-time stability and stabilization of Ito stochastic systems with Markovian switching: mode-dependent parameter approach," Institute of Electrical and Electronics Engineers Transactions on Automatic Control, vol. 60, no. 9, pp. 2428-2433, 2015.

[29] W. Zhang and X. An, "Finite-time control of linear stochastic systems," International Journal of Innovative Computing, Information \& Control, vol. 4, pp. 689-696, 2008.

[30] F. Amato, M. Ariola, and C. Cosentino, "Finite-time control of discrete-time linear systems: Analysis and design conditions," Automatica, vol. 46, no. 5, pp. 919-924, 2010.

[31] J. C. Geromel, P. L. Peres, and J. Bernussou, "On a convex parameter space method for linear control design of uncertain systems," SIAM Journal on Control and Optimization, vol. 29, no. 2, pp. 381-402, 1991. 


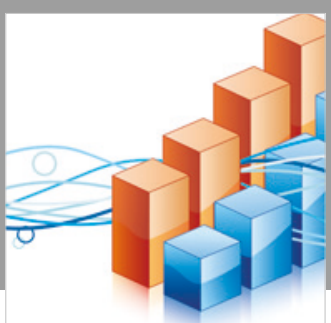

Advances in

Operations Research

\section{-n-m}
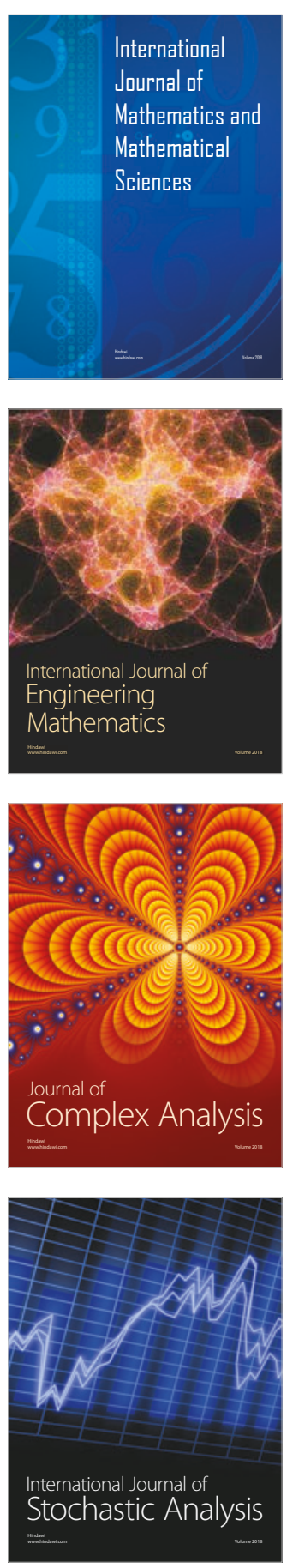
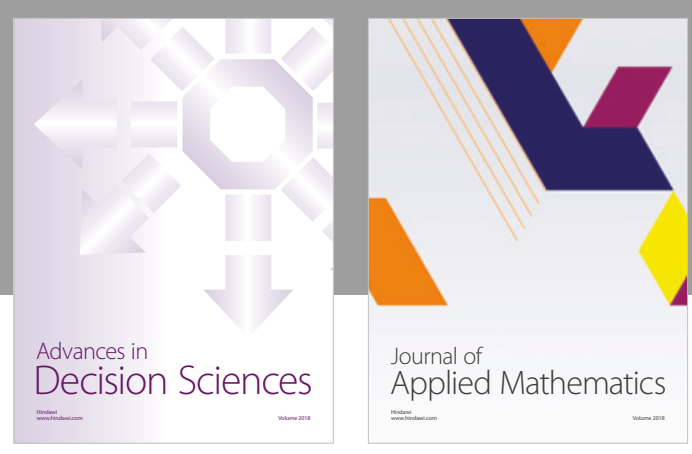

Journal of

Applied Mathematics
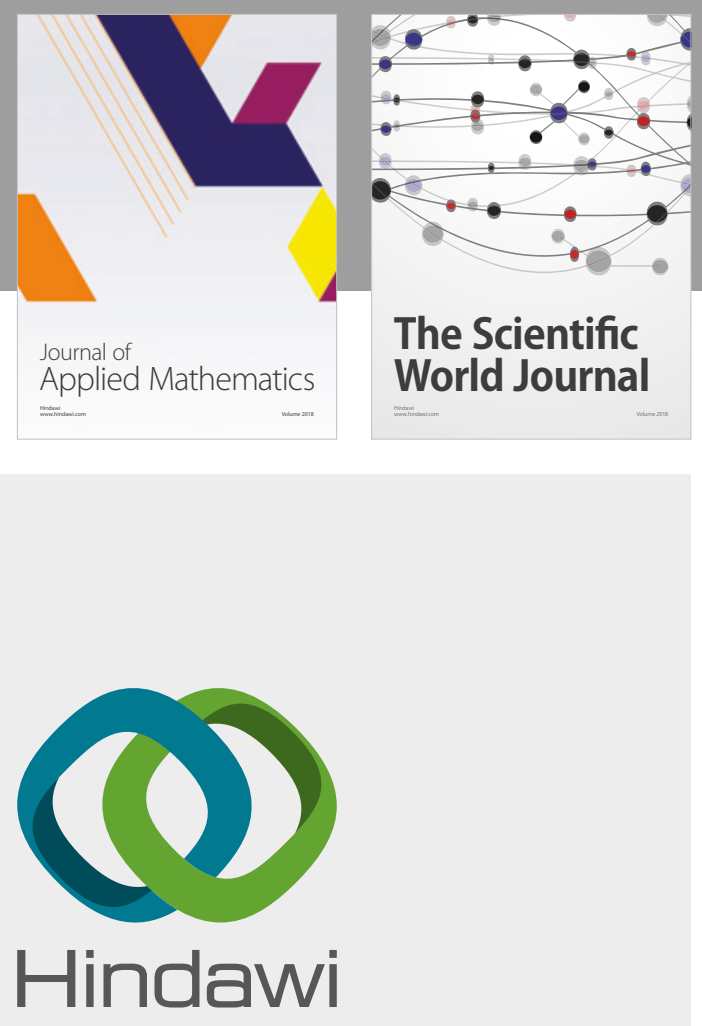

Submit your manuscripts at

www.hindawi.com

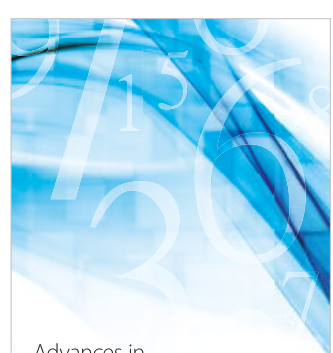

Advances in
Numerical Analysis
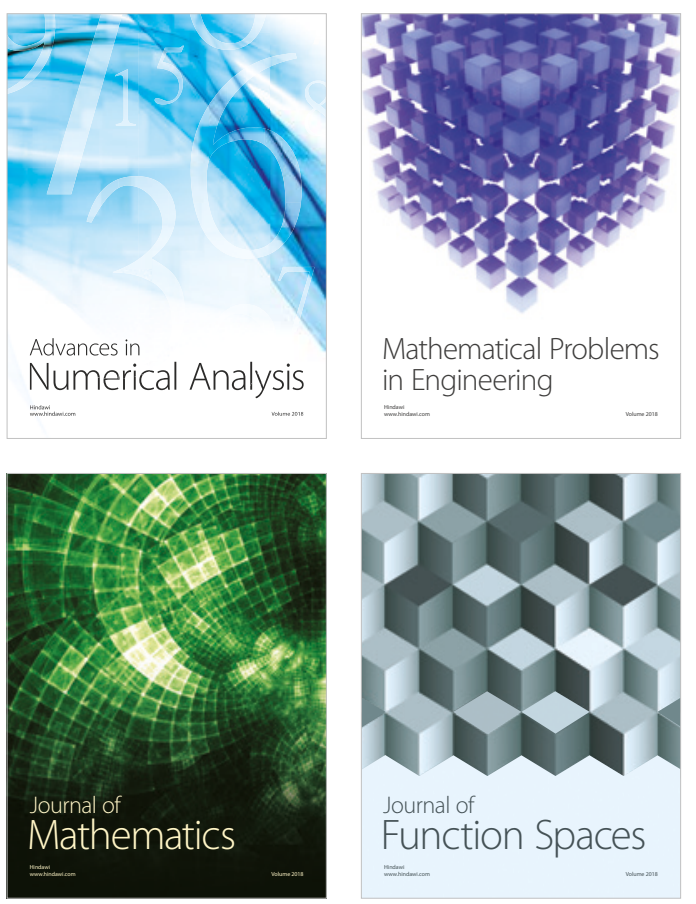

Mathematical Problems in Engineering

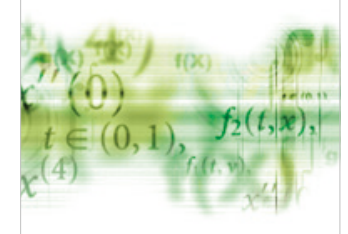

International Journal of

Differential Equations

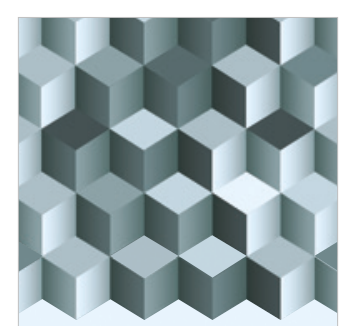

Journal of

Function Spaces
The Scientific

World Journal

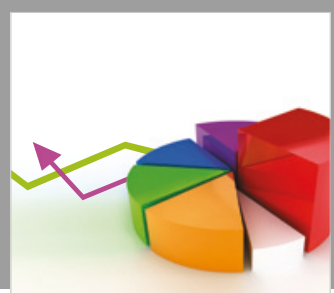

Journal of

Probability and Statistics
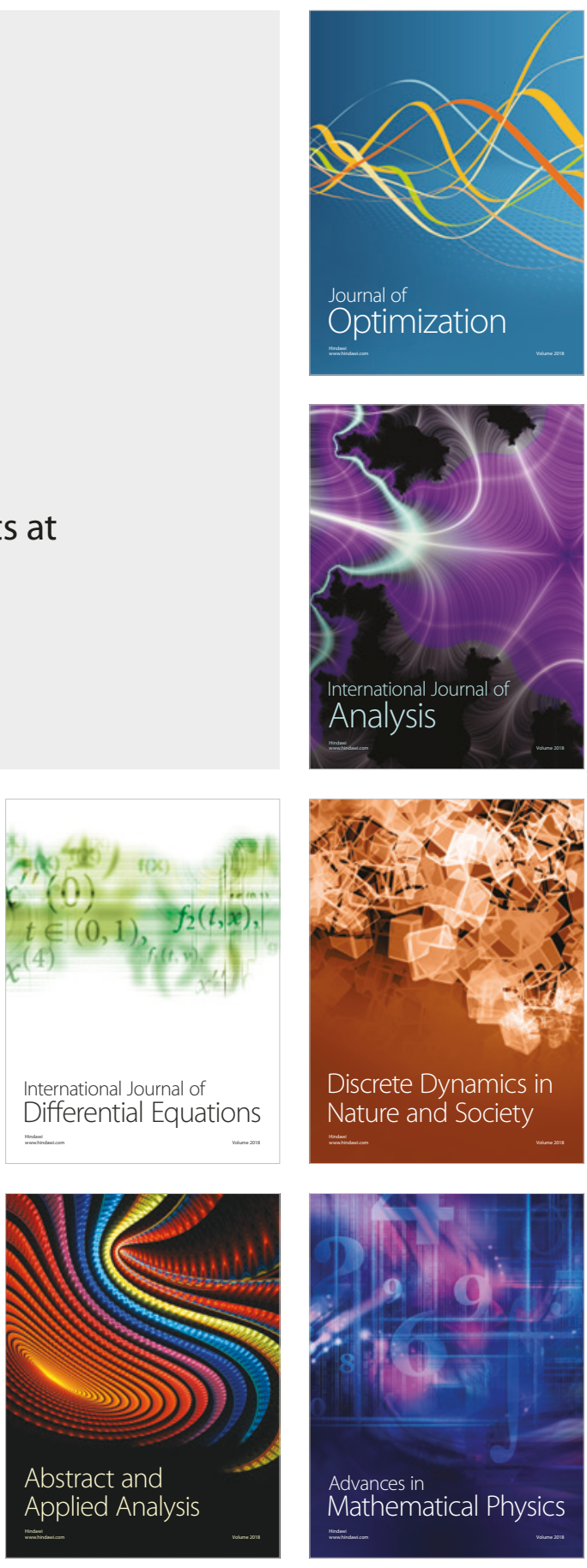